\title{
Enhancing Spectral Efficiency of Transform Domain Communication Systems by using a Multidimensional Modulation
}

\author{
Guillaume Fumat ${ }^{\dagger}$, Pascal Chargé ${ }^{\ddagger}$, Ahmed Zoubir *, Danièle Fournier-Prunaret ${ }^{\dagger}$ \\ ${ }^{\dagger}$ LATTIS - INSA, University of Toulouse, 135 avenue de Rangueil, 31077 Toulouse, FR \\ $\ddagger$ IREENA - Ecole Polytechnique, University of Nantes, BP 50609, 44306 Nantes, FR \\ ${ }^{*}$ Axess Europe, 3 avenue Didier Daurat, 31400 Toulouse, FR \\ Contact's email: guillaume.fumat@insa-toulouse.fr
}

\begin{abstract}
Transform Domain Communication System (TDCS) is a Cognitive Radio technology that has been thoroughly studied by the Air Force Institute of Technology because of its resiliency against jammers or interferences while being noise-like.

However TDCS' huge disadvantage is its low spectral efficiency. In this article, we propose the use of a dense multidimensional modulation to counteract this property. We develop the theoretical Bit Error Rate (BER) performance and show that the theory matches well the simulation.

We point out that this modulation can compete with QPSK systems in terms of spectral efficiency while keeping TDCS' intrinsic assets.
\end{abstract}

\section{INTRODUCTION}

Firstly studied by the Airforce Institute of Technology [13], Transform Domain Communication System (TDCS), is a Cognitive Radio technology that generates interferencefree, noise-like waveforms to avoid wasting unused frequency bands.

However, as shown by the authors in [4], TDCSs suffer from a low spectral efficiency because of their inherent multidimensional nature. To extend its use to higher throughput communications, we propose the use of a multidimensional modulation by using simultaneously $k$ among $N$ dimensions instead of only one at a time, as done in standard Cyclic Shift Keying (CSK) TDCSs. The spectral efficiency is then significantly increased: up to 10 times higher than classical TDCSs. Of course this causes a degradation of BER performance but it still competes with standard PSK systems' performance while keeping the TDCS' advantages.

Before getting into details of the new modulation scheme, we will first start with presenting our TDCS system and recall the spectral efficiency limits that apply. In a third section, we will present our multidimensional modulation with its theoretical performance, and compare then the theory with simulation results. The question of mapping the symbols to the bit words is also covered. In a last section, we will focus on the comparison between the $\left(\begin{array}{l}N \\ k\end{array}\right)$-Ary modulation scheme and the usual PSK modulation scheme before concluding on the applications and limits of this technique.

\section{SyStem MODEL AND ITS DIMENSIONALITY PROPERTY}

\section{A. System Model}

1) Emitter Side: We consider here a CSK-TDCS emitter in Fig. 1. It is however possible to combine CSK and PSK modulation to improve symbols orthogonality (see $[4,6]$ ).

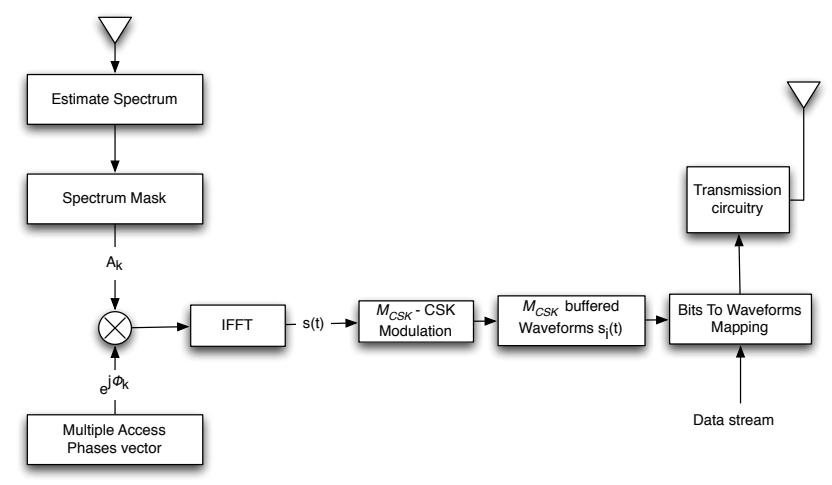

Fig. 1: TDCS Emitter

The first step in a TDCS system consists of sensing the available spectrum to enable interference-free transmissions. Different techniques exist to better detect the interferences [3, 8, 9]. A spectrum mask $S^{(v)}(f)$ of useful bandwidth $W_{u}$ is chosen from this estimate, which the transmitted signal will have to respect. A multiple-access phase $\phi_{r}^{(v)}[1]$ is then added to each of its frequency components and let the signal be noiselike. The resulting spectrum can be written by Eq.(1):

$$
\begin{array}{r}
S^{(v)}(f)=\sum_{r=1}^{K} A_{r}^{(v)} \delta\left(f-r \Delta_{f}\right) e^{+j \phi_{r}^{(v)}}+ \\
A_{r}^{(v)} \delta\left(f+r \Delta_{f}\right) e^{-j \phi_{r}^{(v)}}
\end{array}
$$

with $A_{r} \in\{0,1\}$ the amplitude of the $r$-th frequency component (set by the spectrum sensing), $K$ the number of frequency components, $\Delta_{f}$ the spectral sample spacing and the upper-script $(v)$ denotes the $v$-th user. Defining $K_{\text {used }}$, the number of frequency components of the useful spectrum $A_{r} \neq 0$. We have $W_{u}=K_{\text {used }} \Delta_{f}$. 
The reference waveform is computed from the Inverse Fourier Transform (IFT) of Eq.(1) and the resulting signal can be considered as a symbol whose duration is $T=1 / \Delta_{f}$.

$$
s^{(v)}(t)=2 \sum_{r=1}^{K} A_{r} \cos \left(2 \pi r \Delta_{f} t+\phi_{r}^{(v)}\right)
$$

CSK modulation consists then of cyclically shifting the generated waveform, see Eq.(3). Each shifted waveform represents one symbol (a bit word) and is stored in a buffer.

$$
s_{\mathrm{CSK}_{m_{\mathrm{CSK}}}}(t)=s_{\mathrm{CSK}_{0}}\left(t-\frac{m_{\mathrm{CSK}} T}{M_{\mathrm{CSK}}}\right)_{T}
$$

with $T$ the waveform duration, and $s\left(t-\frac{T}{m}\right)_{T}$ is the notation introduced in [1] for a $\frac{T}{m}$ circular shift. $m_{\mathrm{CSK}} \in$ $\left\{0, \cdots, M_{\mathrm{CSK}}-1\right\}$ is the CSK symbol index.

The set of shifted waveforms $s_{\mathrm{CSK}_{0}} \cdots s_{\mathrm{CSK}_{\left(M_{\mathrm{CSK}}-1\right)}}$ can be considered as pseudo-orthogonal [10]. We then sum $k$ waveforms from the $N$ buffered ones and transmit the resulting symbol. In standard CSK modulation, only one waveform corresponds to one bit word $(k=1)$. Summing $k$ waveforms leads to a dense multidimensional constellation with a higher spectral efficiency.

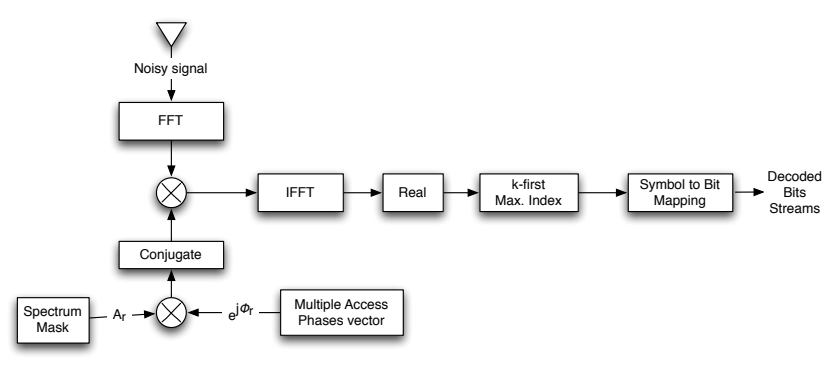

Fig. 2: TDCS Optimum Receiver

2) Receiver Side: In Fig. 2, we assume that the receiver and the emitter share the same spectrum mask, random phases and mapping between a waveform and a bit word. The demodulation process described in Fig. 2 is equivalent to an optimum AWGN demodulator [5, 10]. By taking the $k$-first maximum indexes one knows which symbol is incoming and demapping into a bits stream is then achieved. The demapping process is further detailed in Section III-B.

3) System Parameters: In our TDCS system, the signal's bandwidth is defined from $1 \mathrm{kHz}$ to $7 \mathrm{MHz}$ and avoids interferences between 2 and $3 \mathrm{MHz}$. As a consequence, its total available bandwidth is $W_{a}=6.999 \mathrm{MHz}$, and its used bandwidth is $W_{u}=5.999 \mathrm{MHz}$, described by $K_{\text {used }}=256$ frequency components. The dimensionality of our system is $N=512$. These figures are summarized in the spectrum mask of Fig. 3

Now that our TDCS system model is introduced, let us see the role that plays the dimensionality parameter $N$ on the system's performance.

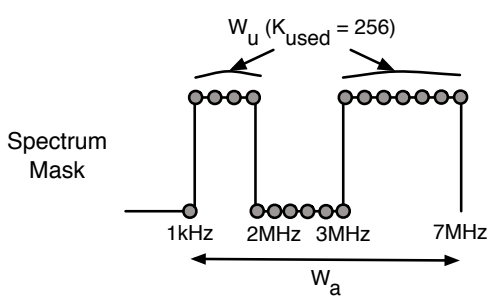

Fig. 3: Signal's Spectrum

\section{B. Impacts of Dimensionality on Spectrum Efficiency}

Let us recall the dimensionality $N$ of a signal $x(t)$ that roughly lasts $T$ seconds and occupies a bandwidth $W_{u}$. It defines the number of orthogonal signals such that $x(t)$ can be expressed by a linear combination of them. In [7, pp 227229], it is stated that $N$ is well approximated by Eq. (4):

$$
N \approx 2 W_{u} T
$$

More specifically for a TDCS system, since the period symbol is $T=\frac{1}{\Delta_{f}}$, and since $W_{u}=K_{\text {used }} \Delta_{f}$, the signal dimensionality is equal to $N=2 K_{\text {used }}$.

As a consequence, one can write the spectrum efficiency $\eta_{\text {eff }}$ of a multidimensional system as it follows in Eq.5:

$$
\begin{aligned}
\eta_{\mathrm{eff}} & =\frac{R_{b i t}}{W_{u}}=\frac{\log _{2}\left(M_{m o d}\right) R_{s y m b}}{\frac{N}{2 T}} \\
& =\frac{\log _{2}\left(M_{\text {mod }}\right)}{K_{\text {used }}}
\end{aligned}
$$

where $M_{\text {mod }}$ is the CSK constellation size, $R_{b i t}$ is the bit-rate, and $R_{\text {symb }}=\frac{R_{b i t}}{\log _{2}\left(M_{m o d}\right)}=\frac{1}{T}$ is the symbol rate.

As a consequence, TDCSs with a high dimensionality suffer from a low spectral efficiency. To improve the latter, it is necessary to increase $M_{\text {mod }}$. In the following, we use the modulation presented in Section II-A with $k>1$.

\section{III. $\left(\begin{array}{l}N \\ k\end{array}\right)$-ARY MODUlatION FOR TDCS}

CSK is considered as a standard M-ary Orthogonal Signaling (MOS) [10] with only one dimension used per symbol. We propose here to represent a symbol by an arrangement of $k$ dimensions that results in having $M_{\bmod }=\left(\begin{array}{c}N \\ k\end{array}\right)$ possible symbols. It produces thereby an important increase in the spectral efficiency. We will now quantify this increase and compute the BER performance that this modulation can reach.

\section{A. Theoretical Performance}

1) Maximum Spectral Efficiency: It is straightforward to note that the constellation size $\left(\begin{array}{l}N \\ k\end{array}\right)$ reaches a maximum for $k=\frac{N}{2}$. Thus, because of Eq.(5), we can infer the maximum spectral efficiency of the modulation in Eq. (6):

$$
\eta_{\mathrm{eff}_{\max }}=\frac{2 \log _{2}\left(\begin{array}{l}
N \\
\frac{N}{2}
\end{array}\right)}{N}
$$

By means of the Stirling's approximation given in Eq.(7), it is possible to deduce $\eta_{\text {eff }_{\max }}$ in Eq.(8). 


$$
\begin{aligned}
\log _{2}(N !) \approx & N\left(\log _{2} N-\frac{1}{\ln 2}\right)+\frac{1}{2} \log _{2}(N)+\frac{1}{2} \log _{2} 2 \pi \\
\eta_{\mathrm{eff}_{\max }} & =\frac{2 \log _{2}\left(\begin{array}{l}
N \\
\frac{N}{2}
\end{array}\right)}{N}=2\left[\frac{\log _{2} N !}{N}-\frac{\log _{2}\left(\frac{N}{2} !\right)}{\frac{N}{2}}\right] \\
& \approx 2\left[1+\frac{1-\log _{2} 2 \pi-\log _{2} N}{N}\right] \\
\lim _{N \rightarrow \infty} \eta_{\mathrm{eff}_{\max }} & =2
\end{aligned}
$$

According to Eq.(8), $\eta_{\mathrm{eff}_{\max }}$ converges slowly to 2 . For example, $N=32$ can provide spectral efficiency of $1.82 \mathrm{bits} / \mathrm{s} / \mathrm{Hz}$, close to the QPSK spectral efficiency. Using higher $N$ would lead to a significant increase in the system's complexity while providing only a tenth increase in the spectral efficiency.

2) BER Performance: We base our approach on the same formalism as in [7]. Thus, symbols of our $\left(\begin{array}{l}N \\ k\end{array}\right)$-Ary modulation, can be written as in Eq.(9):

$$
\begin{aligned}
\mathbf{s}_{1} & =\overbrace{\left.\sqrt{\frac{\epsilon}{k}}, \cdots, \sqrt{\frac{\epsilon}{k}}, 0 \cdots, 0\right)}^{k} \\
\cdots & \\
\mathbf{s}_{i} & =\left(0, \sqrt{\frac{\epsilon}{k}}, \sqrt{\frac{\epsilon}{k}}, 0, \cdots, \sqrt{\frac{\epsilon}{k}} \cdots, 0\right)
\end{aligned}
$$

with $i \in I=\left\{1 \cdots\left(\begin{array}{l}N \\ k\end{array}\right)\right\}$, and $\epsilon$ the symbol energy.

In the context of CSK-based TDCS, sending the symbol $s_{i}$ corresponds to send the waveform $s_{i}(t)$ defined in Eq.(10):

$$
s_{i}(t)=\sum_{m=1}^{N}\left[\mathbf{s}_{i}\right]_{m} s_{\mathrm{CSK}_{0}}\left(t-\frac{m T}{N}\right)_{T}
$$

with $\left[\mathbf{s}_{i}\right]_{m}$ equals the $m$-th component of symbol $\mathbf{s}_{i}$ being sent (see Eq. (9)).

We develop now the analytical BER of a $\left(\begin{array}{l}N \\ 2\end{array}\right)$-Ary modulation but the following development is similar for any $k$. Without loosing generality, suppose the the symbol $s_{1}$ is sent:

$$
\mathbf{s}_{1}=\left(\sqrt{\frac{\epsilon}{2}}, \sqrt{\frac{\epsilon}{2}}, 0, \cdots, 0\right)
$$

Symbol $\mathbf{s}_{1}$ is now corrupted by an AWGN noise $\mathbf{n}$ :

$$
\mathbf{n}=\left(n_{1}, n_{2} \cdots, n_{N}\right)
$$

with $n_{i}, i \in\{1 \cdots N\}$, are zero-mean, stastically independent Gaussian random variables, each of variance $\frac{N_{0}}{2}$. The received signal can then be written as :

$$
\mathbf{r}_{1}=\left(\sqrt{\frac{\epsilon}{2}}+n_{1}, \sqrt{\frac{\epsilon}{2}}+n_{2}, n_{3}, \cdots, n_{N}\right)
$$

To achieve an optimum demodulation [7], there needs to be a correlation between $\mathbf{r}_{1}$ and the $\left(\begin{array}{c}N \\ 2\end{array}\right)$ possible symbols $\mathbf{s}_{p}$, $p \in I$. Among these correlations, there are 4 different cases listed in Eq.(14).

$$
\begin{aligned}
& \mathbf{r}_{1} \cdot \mathbf{s}_{1}=\epsilon+n_{1} \sqrt{\frac{\epsilon}{2}}+n_{2} \sqrt{\frac{\epsilon}{2}} \\
& \mathbf{r}_{1} \cdot \mathbf{s}_{a}=\frac{\epsilon}{2}+n_{1} \sqrt{\frac{\epsilon}{2}}+n_{i} \sqrt{\frac{\epsilon}{2}} \\
& \mathbf{r}_{1} \cdot \mathbf{s}_{b}=\frac{\epsilon}{2}+n_{2} \sqrt{\frac{\epsilon}{2}}+n_{j} \sqrt{\frac{\epsilon}{2}} \\
& \mathbf{r}_{1} \cdot \mathbf{s}_{c}=n_{k_{1}} \sqrt{\frac{\epsilon}{2}}+n_{k_{2}} \sqrt{\frac{\epsilon}{2}}
\end{aligned}
$$

with $a, b, c$ different symbol indexes belonging to disjoint sets $A, B, C$ forming a partition of $I$. Index sets $A$ and $B$ are of size $N-2$, and $C$ is of size $\left(\begin{array}{c}N-2 \\ 2\end{array}\right) . i, j \in\{3 \cdots N\}$, $\left(k_{1}, k_{2}\right) \in\{3 \cdots N\}^{2}$ with $k_{1} \neq k_{2}$.

The probability of correctly receiving the symbol $\mathbf{s}_{1}$ is the probability given in (15) :

$$
\begin{aligned}
P_{c}= & P\left[\bigcap_{a \in A}\left(\mathbf{r}_{1} \cdot \mathbf{s}_{1}>\mathbf{r}_{a} \cdot \mathbf{s}_{a}\right), \bigcap_{b \in B}\left(\mathbf{r}_{1} \cdot \mathbf{s}_{1}>\mathbf{r}_{b} \cdot \mathbf{s}_{b}\right),\right. \\
& \left.\bigcap_{c \in C}\left(\mathbf{r}_{1} \cdot \mathbf{s}_{1}>\mathbf{r}_{c} \cdot \mathbf{s}_{c}\right)\right] \\
P_{c}=P & {\left[\bigcap_{i=3}^{N}\left(\sqrt{\frac{\epsilon}{2}}+n_{1}>n_{i}\right), \bigcap_{j=3}^{N}\left(\sqrt{\frac{\epsilon}{2}}+n_{2}>n_{j}\right),\right.} \\
& \left.\bigcap_{\substack{k_{1}=3 . . N \\
k_{2}=3 . . N \\
k_{1} \neq k_{2}}}\left(\sqrt{2 \epsilon}+n_{2}+n_{1}>n_{k_{1}}+n_{k_{2}}\right)\right]
\end{aligned}
$$

We can note that each event is not independent. A first step in simplifying the formula is to condition the probability of Eq.(15) on $n_{1}$ and $n_{2}$. This gives Eq.(16)

$$
\begin{aligned}
P_{c}= & \iint_{\mathbb{R}^{2}} P\left[\bigcap_{i=3}^{N}\left(\sqrt{\frac{\epsilon}{2}}+n>n_{i}\right), \bigcap_{j=3}^{N}\left(\sqrt{\frac{\epsilon}{2}}+n^{\prime}>n_{j}\right),\right. \\
& \left.\bigcap_{\substack{k_{1}=3 . . N \\
k_{2}=3 . . N \\
k_{1} \neq k_{2}}}\left(\sqrt{2 \epsilon}+n+n^{\prime}>n_{k_{1}}+n_{k_{2}}\right)\right] e^{-\frac{n^{2}}{N_{0}}} e^{-\frac{n^{\prime 2}}{N_{0}}} \\
& \frac{1}{\pi N_{0}} d n d n^{\prime}
\end{aligned}
$$

However, there is still a coupling between the intersections. But since one event $\left\{\sqrt{\frac{\epsilon}{2}}+n>n_{i}\right\}$ is coupled to three other ones, while it is independent with $\left(\left(\begin{array}{c}N \\ 2\end{array}\right)-4\right)$ other events, we do the approximation in Eq.(17) that every event is independent. We will see in Section III-C, that this approximation is reasonable. 


$$
\begin{aligned}
P_{c} \approx & \iint_{\mathbb{R}^{2}}\left(1-\mathcal{Q}\left(\frac{n+\sqrt{\frac{\epsilon}{2}}}{\sqrt{N_{0} / 2}}\right)\right)^{N-2}\left(1-\mathcal{Q}\left(\frac{n^{\prime}+\sqrt{\frac{\epsilon}{2}}}{\sqrt{N_{0} / 2}}\right)\right)^{N-2} \\
& \left(1-\mathcal{Q}\left(\frac{n+n^{\prime}+\sqrt{2 \epsilon}}{\sqrt{N_{0}}}\right)\right)^{\left(\begin{array}{c}
N-2 \\
2
\end{array}\right)} e^{-\frac{n^{2}}{N_{0}}} e^{-\frac{n^{\prime 2}}{N_{0}}} \frac{1}{\pi N_{0}} d n d n^{\prime}
\end{aligned}
$$

Taking $u=\frac{n+\sqrt{\frac{\epsilon}{2}}}{\sqrt{N_{0} / 2}}$, and $v=\frac{n^{\prime}+\sqrt{\frac{\epsilon}{2}}}{\sqrt{N_{0} / 2}}$, we can express the symbol error probability as Eq.(18) :

$$
\begin{gathered}
P_{e}=1-\iint_{\mathbb{R}^{2}} \frac{1}{2 \pi}(1-Q(u))^{N-2}(1-Q(v))^{N-2} \\
\quad\left(1-Q\left(\frac{u+v}{\sqrt{2}}\right)\right)^{\left(\begin{array}{c}
N-2 \\
2
\end{array}\right)} e^{\left.-\frac{\left(u-\sqrt{\frac{\epsilon}{N_{0}}}\right.}{2}\right)^{2}} e^{-\frac{\left(v-\sqrt{\frac{\epsilon}{N_{0}}}\right)^{2}}{2}} d u d v
\end{gathered}
$$

Symbol Error Rate (SER) of Eq. (18) can be computed numerically. In a very similar way, performance of a $\left(\begin{array}{c}N \\ 3\end{array}\right)$ modulation can also be derived. ${ }^{1}$ It is however complicated to develop a bit error probability since it highly depends on the mapping between the bit words and the symbols. But as a first approximation, considering large $N,\left(\begin{array}{c}N-2 \\ 2\end{array}\right) \gg 2(N-2)$, we can assume that every symbol has the same inter-symbol distance $d=2 \sqrt{\epsilon}$. Which is also the case for standard MOS. As a matter of fact, we can similarly approximate [7, p. 205]:

$$
P_{b}=\frac{2^{k-1}}{2^{k}-1} P_{e}
$$

As previously raised, constellation size such $M=\left(\begin{array}{c}128 \\ 3\end{array}\right)=$ 341376 makes obvious the problem of mapping such a high number of symbols to a bit word. We propose a solution in the next subsection.

\section{B. Symbol to Bit words Mapping and vice versa}

We propose here to map a huge number of symbols to bit words, not in an optimum way, but in a computation-efficient way. We base our algorithm on a straightforward way to order the different $\left(\begin{array}{l}N \\ k\end{array}\right)$ combinations. For example with $M=\left(\begin{array}{l}5 \\ 3\end{array}\right)$ we have the exhaustive list of combinations in table I.

$d_{1}, d_{2}$ and $d_{3}$ represent the dimension indexes. Because this order is very structured, it is easy to find out an algorithm that associates the combination indexes to the dimension indexes. Taking the binary value of the combination index gives a mapping between bit words and waveforms. ${ }^{1}$

\section{Simulation Results}

To validate the theory developed above for $k \in\{2,3\}$, we used orthogonal waveforms generated by CSK and chose a modulation using only 32 dimensions from 512 to ensure orthogonality.

\footnotetext{
${ }^{1}$ To ensure paper's brevity, the authors decided not to detail these algorithms or computations.
}

\begin{tabular}{|r||ccc|}
\hline \multicolumn{1}{|r||}{ Index } & $d_{1}$ & $d_{2}$ & $d_{3}$ \\
\hline \hline 1 & 1 & 2 & 3 \\
\hline 2 & 1 & 2 & 4 \\
\hline 3 & 1 & 2 & 5 \\
\hline 4 & 1 & 3 & 4 \\
\hline 5 & 1 & 3 & 5 \\
\hline 6 & 1 & 4 & 5 \\
\hline 7 & 2 & 3 & 4 \\
\hline 8 & 2 & 3 & 5 \\
\hline 9 & 2 & 4 & 5 \\
\hline 10 & 3 & 4 & 5 \\
\hline
\end{tabular}

TABLE I: Every combination of 3 dimensions among 5
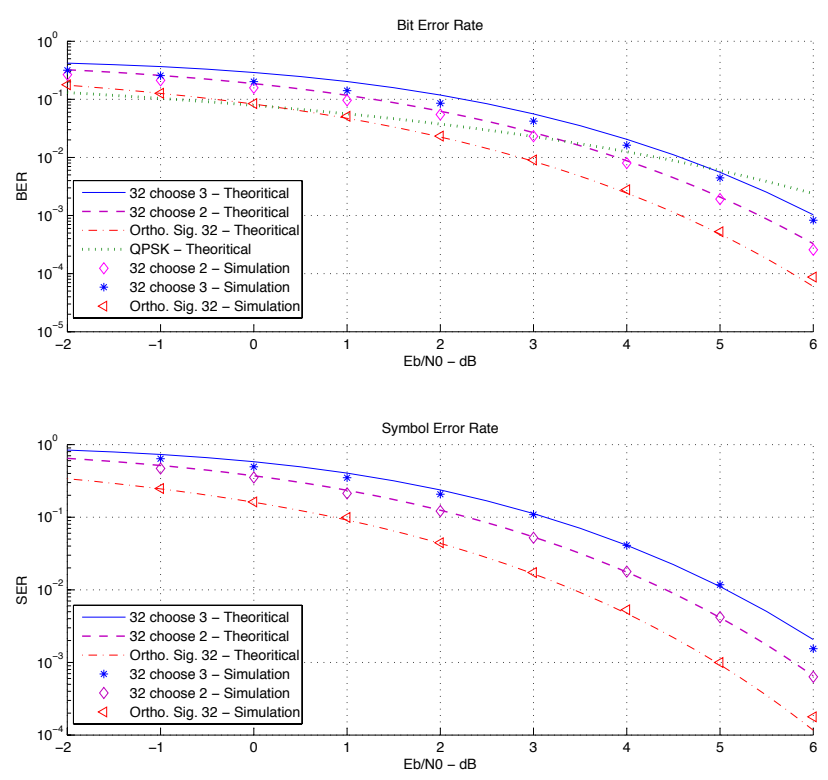

Fig. 4: SER and BER performance of $\left(\begin{array}{c}32 \\ 2\end{array}\right)$ and $\left(\begin{array}{c}32 \\ 3\end{array}\right)$ modulation

First of all we observe on Fig. 4 that the simulated and theoretical SER match in spite of our approximation. As expected, $\left(\begin{array}{c}32 \\ 3\end{array}\right)$ modulation performs worse than $\left(\begin{array}{c}32 \\ 2\end{array}\right)$, which itself shows worse performance than standard CSK modulation.

However concerning the BER performance, the simulated curves are below the theoretical ones for low $E_{b} / N_{0}$ values. This is due to our assumption that a symbol error causes half of its bits to be wrong : the worst case scenario. We can thus infer that our computation-efficient mapping performs better than the worst case mapping.

If we consider now a system using 32 dimensions generated by CSK from only 64 theoretically available, the orthogonality property of the symbols set is strongly affected as shown in table II. This results in a slight gap between the theory and the simulation of MOS at a BER of $1 \times 10^{-4}$. However, this difference becomes much larger when using the $\left(\begin{array}{c}32 \\ 2\end{array}\right)$ modulation as highlighted on Fig. 5.

Although showing worse BER performance than MOS, we can see on Fig. 4 that $\left(\begin{array}{c}N \\ k\end{array}\right)$-Ary modulation remains better than standard QPSK performance for some $E_{b} / N_{0}$ range. The question that follows is "When does the $\left(\begin{array}{l}N \\ k\end{array}\right)$ modulation provides better BER than PSK at the same spectral efficiency?". 


\begin{tabular}{|r||c|c|}
\hline Statistics & $\mathrm{N}=64$ & $\mathrm{~N}=512$ \\
\hline \hline Mean & 0.001 & -.004 \\
\hline Min & -.21 & -.029 \\
\hline Max & 0.32 & .0048 \\
\hline Var & 0.01 & $7.42 \times 10^{-5}$ \\
\hline
\end{tabular}

TABLE II: Scalar Product Statistics between 32-CSK waveforms for different dimensionality value

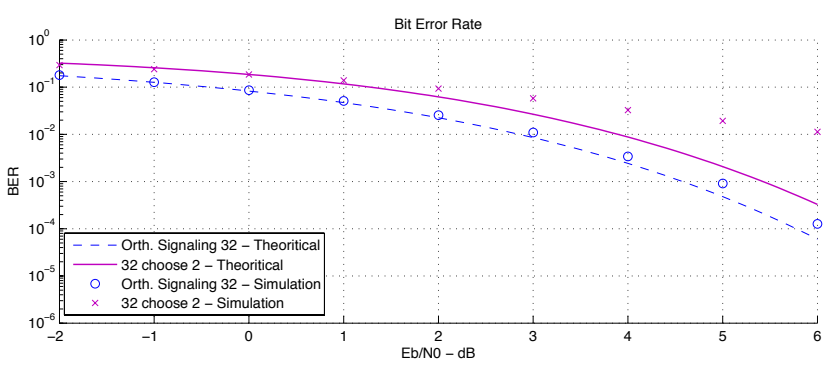

Fig. 5: $\left(\begin{array}{c}32 \\ 2\end{array}\right)$ modulation with insufficient orthogonality

\section{COMPARISON BETWEen $\left(\begin{array}{l}N \\ k\end{array}\right)$-ARY MOdUlation AND PSK MODULATION}

As previously done, we assume the use of purely orthogonal waveforms.

As seen in Section III-A, the computation of the theoretical performance of a $\left(\begin{array}{c}N \\ k\end{array}\right)$-Ary modulation system, requires $k$ infinite integrals. Thus, the results presented here are from MonteCarlo simulations for the $\left(\begin{array}{c}N \\ k\end{array}\right)$-Ary modulation, theoretical BER curves are taken for PSK. We then plot in Fig.6 their spectral efficiency in function of the $E_{b} / N_{0}$ while ensuring $1 \times 10^{-3}$ and $1 \times 10^{-5}$ BER goals.

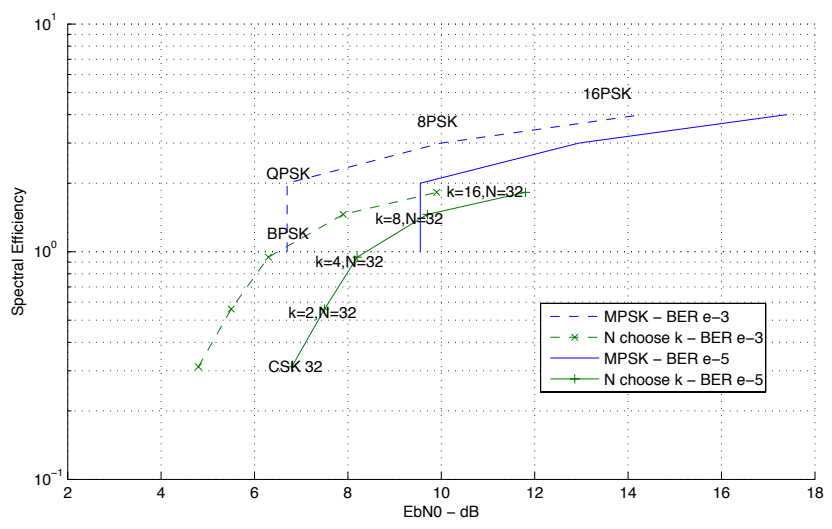

Fig. 6: Spectral Efficiency comparison for 2 BER goals

Each dot represents a modulation scheme, varying from BPSK to 16 PSK and from $k=1$ to $k=16$ for respectively PSK and $\left(\begin{array}{c}32 \\ k\end{array}\right)$-Ary modulations.

First we note that small values of $k$ let the system achieve the BER goal for low $E_{b} / N_{0}$, while PSK cannot. Then for $k=8$, a spectral efficiency of $1.6 \mathrm{bits} / \mathrm{sec} / \mathrm{Hz}$ is achieved much after the PSK systems when the BER goal is $1 \times 10^{-3}$, but at the same $E_{b} / N_{0}$ than PSK systems for a BER goal of $1 \times$ $10^{-5}$. This is due to the BER performance curves of $\left(\begin{array}{l}N \\ k\end{array}\right)$-Ary modulation: much steeper than the PSK ones. As a result, when decreasing the BER goal, spectral efficiency of both systems suffer from a translation to higher $E_{b} / N_{0}$, but this translation is narrower for $\left(\begin{array}{c}N \\ k\end{array}\right)$-Ary modulation than for PSK. We can expect that this modulation scheme performs better than QPSK for some very low BER goal.

Hence, the proposed modulation scheme has a wide range of working conditions, from low spectral efficiency transmissions at very low $E_{b} / N_{0}$, up to high throughput transmissions at high $E_{b} / N_{0}$. However, there is a tradeoff to do between orthogonality and system complexity : CSK provides only pseudo-orthogonal waveforms but at a low complexity cost. Purely orthogonal waveforms would provide better results.

\section{COnClusion And Future Works}

In this paper, a dense multidimensional constellation modulation scheme is developed and applied to Transform Domain Communication Systems. Its analytical performance are derived and simulations confirm the theory. TDCS' spectral efficiency is increased while accepting a decrease in BER performance. We pointed out that this technique can compete with QPSK spectral efficiency when the BER goal is low, while keeping TDCS's assets. As a consequence the modulation allows a wide range of working condition and can be seen as a new degree of adaptive modulation.

Future work to enhance the $\left(\begin{array}{c}N \\ k\end{array}\right)$-Ary modulation includes the search of optimum mapping and a better orthogonal waveforms generation, since it appears CSK reaches its limits for higher constellation size.

\section{REFERENCES}

[1] P. J. Swackhammer, "Performance Simulation of a Transform Domain Communication System for Multiple Access Applications", MILCOM'99 , pp. 1055-1059 Vol.2, October 1999

[2] A. S. Nunez, M.A. Temple, R.F. Mills, and R.A. Raines, "Interference Avoidance in Spectrally Encoded Multiple Access Communications Using MPSK Modulation", WCNC 2005 , pp. 730-734 Vol.2, March 2005

[3] M. J. Lee, M.A. Temple, R.L. Claypoole, and R.A. Raines, "Interference avoidance communications using wavelet domain transformation techniques", WCNC2002 , pp. 255-259 , March 2002

[4] G. Fumat, P. Chargé , A. Zoubir, D. Fournier-Prunaret, "Transform Domain Communication Systems from a multidimensional perspective, impacts on bit error rate and spectrum efficiency", IET Communications, vol. 5, Issue 4, pp. 476-483, March 2011

[5] C. Han, J. Wang, S. Gong, and S. Li "Adressing the control channel design problem : OFDM-based transform domain communication system in cognitive radio", Computer Networks 2008 vol. 52, n.4, pp. 795-815, June 2008

[6] I. Budiarjo, H. Nikookar, L.P. Lighthart, "On the Utilization of Embedded Symbol for CCSK BER Improvement in TDCS Dynamic Spectrum Access", EuWiT 2008 , pp. 123-126, October 2008

[7] J. G. Proakis, Masoud Salehi Digital Communications, Fifth Edition McGraw-Hill, 2008

[8] C. Wang, Z. Zhang, S. Li, Q. Fu, "Transform Domain Communication System Based on Random-Ambiguity and Fractional Fourier Transform", ITS Telecommunications Proceedings 2006 , pp. 194-197, June 2006

[9] J-Y. Liu, Y.T. Su, "Performance Analysis of Transform Domain Communication Systems in the Presence of Spectral Mismatches", MILCOM 2007 , pp. 1-5, October 2007

[10] G. M. Dillard, M. Reuter, J. Zeidler, B. Zeidler, "Cyclic code shift keying: a low probability of intercept communication technique", IEEE Transactions on Aerospace and Electronic Systems Vol. 39, Issue 3, pp. 786-798, July 2003 